\title{
Bifurcation analysis and nonlinear dynamics of a capacitive energy harvester in the vicinity of the primary and secondary resonances
}

\author{
Saber Azizi ${ }^{1}$, Hadi Madinei²*, Javad Taghipour ${ }^{2}$, Hassen M. Oukad ${ }^{3}$ \\ ${ }^{1}$ Urmia University of Technology, Urmia, Iran \\ ${ }^{2}$ Faculty of science and Engineering, Swansea University, Swansea, UK \\ ${ }^{3}$ Mechanical and Industrial Engineering Department, Sultan Qaboos University, Muscat, Oman
}

\begin{abstract}
The impetus of the present study is to examine the effect of nonlinearity on the efficiency enhancement of a capacitive energy harvester. The model consists of a cantilever microbeam underneath which there is an electret layer with a surface voltage, which is responsible for the driving energy. The packaged device is exposed to unwanted harmonic mechanical excitation. The microbeam undergoes mechanical vibration and accordingly the energy is harvested throughout the output circuit. The dynamic formulation accounts for nonlinear curvature, inertia, and nonlinear electrostatic force. The efficiency of the device in the vicinity of the primary and super-harmonic resonances is examined and accordingly the output power is evaluated. Bifurcation analysis is carried out on the dynamics of the system by detecting the bifurcations in the frequency domain and diagnosing their types. One of the challenging issues in the design and analysis of energy harvesting devices is to broaden the bandwidth so that more frequencies are accommodated within the amplification region. In this study the effect of the nonlinearity on the bandwidth broadening, as well as efficiency improvement of the device, is studied.
\end{abstract}

Key Words: Capacitance; Energy Harvesting device; Non-linearity; Cantilever; MEMS.

\section{Introduction}

Wireless sensor networks (WSN) have been focused in recent years due to their numerous advantages such as ease of installation, reduction in cost and weight, and elimination of wire connections [1]. Providing the power supply source is a challenge issue in these systems. Batteries provide an impermanent power supply solution which it takes lots of time and cost to replace them. An interesting and long-term solution to extend the operational time of WSNs is vibration energy harvesting devices which convert the ambient vibrations into electrical energy to charge the batteries or replacing them. In the past few years many researchers have focused on the dynamics of energy harvesting devices so that to enhance the efficiency of the energy harvester [1-3]. Among the numerous publications reported so

* Corresponding author.

E-mail address: hadi.madinei@swansea.ac.uk 
far, some of them have focused on harvesting energy based on electromagnetic effect [4-9], some have focused on piezoelectric based ones [1,2,10-14]. Capacitive energy harvesting devices have also been focused in the literature thanks to their ease of fabrication; however, as a major challenge, they require a voltage source to induce electric charges on the electrodes of the variable capacitor and consequently, start the conversion [3, 15-18]. The required voltage can be provided by means of using dielectric, capable of keeping internal charges for many years. Some researchers have explored the possibility of amplitude enhancement thanks to the internal resonance [14, 19, 20]. A very important research subject which has received a great research interest, is the effect of nonlinearity on the performance and the efficiency of an energy harvesting devices. The findings of the most important of these are reviewed here. Ngan Tran et al. [21] reported a review on nonlinear techniques for performance enhancement of ambient vibration of energy harvesters. They reported the most important publications devoted to the effect of stochastic loading, internal resonance, being multi-degree of freedom, mechanical stoppers and parametric excitation, which lead to nonlinear behaviour and accordingly enhance the efficiency of the energy harvesting device. Lu et al. [22] explored the connection between the resonance response interaction and bubble shaped response curve that can occur in the forced response of a nonlinear magnetoelectric coupled system. Hao $\mathrm{Wu}$ et al. [23] proposed a nonlinear two degree of freedom piezoelectric energy harvester based on the similar linear model proposed by $\mathrm{Wu}$ et al. [24]; they achieved broader operating bandwidth in mono-stable condition by taking benefit of the nonlinearity and also tuning the resonance response peaks in the frequency domain. A Cammarano et al. [25] improved the band width of an energy harvester exploiting nonlinearities. They reported that the stronger is the nonlinearity, the broader is the band width and accordingly the better is the performance; this is due to the fact that more frequency contents can be accommodated in the band width.

Based on the literature, one of the main issues with the vibrating energy harvesters, is the narrow bandwidth in case of high quality factor resonators [21]; the impetus of the current research is to broaden the bandwidth of a capacitive energy harvesting device exposed to unwanted harmonic external excitations, thanks to the existence of nonlinearity. The effect of nonlinearities including geometric, inertia and nonlinear displacement dependent electrostatic force, on the efficiency of the device are taken into account. The study encompasses the response in the vicinity of both primary and super-harmonic resonances. The frequency response curves are computed by means of Shooting technique and the stability of the periodic solutions are ascertained based on Floquet theory. The bifurcations in the frequency domain are determined and their types are examined based on the loci of the Floquet exponents with respect to the unit circle.

\section{Model description and mathematical modelling}

As depicted in Fig. 1, the proposed energy harvester is composed of a cantilever beam exposed to underneath capacitance. Between the lower electrode and the cantilever beam, exists an electret layer with a surface voltage of $V_{s}$, thickness of $h_{e}$, and relative permittivity of $\varepsilon_{e}$, which is responsible for providing the required voltage of the device. 


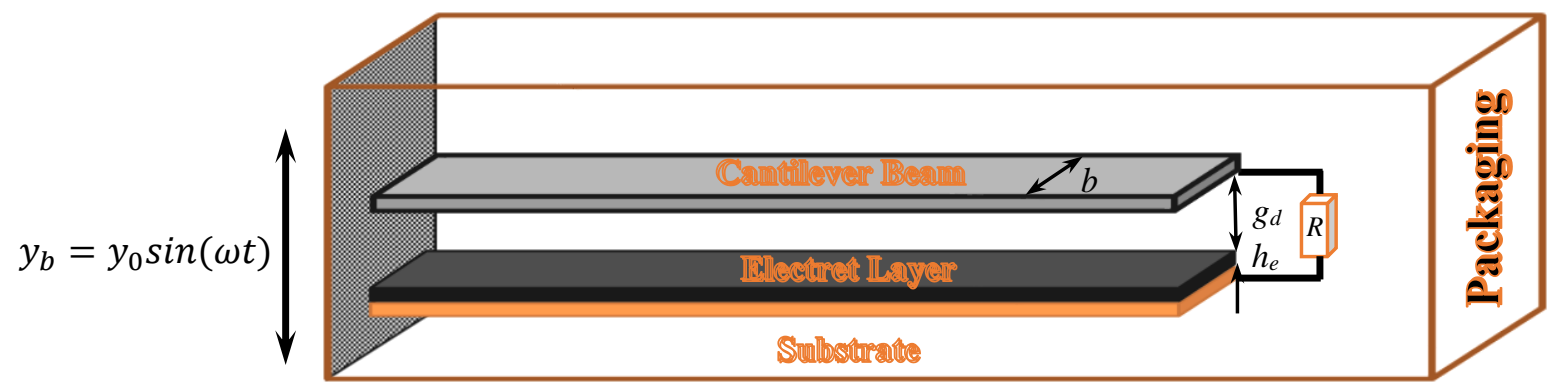

Fig. 1 Schematics of the proposed energy harvester

In order to model the mechanical behavior of the micro-cantilever, an Euler-Bernoulli beam theory is utilized. The coordinate system $(x-y-z)$ is attached to the fixed end of the cantilever beam with its origin on the neutral axis and the $x$ axis along the beam and the $y$ axis in the direction of the base excitation. Assuming the energy harvester operating as a high-quality factor resonator and accordingly undergoing large deformations, the geometrical and inertial nonlinearities due to inextensionality conditions get more pronounced and the linear theory does not hold true anymore. Therefore, a full nonlinear beam model is employed which takes the curvature nonlinearities and shortening effects into account. The nonlinear equation of motion for the considered micro cantilever based on the Euler-Bernoulli beam theory is given by [26],

$$
\begin{gathered}
\rho A \frac{\partial^{2} w}{\partial t^{2}}+E I \frac{\partial}{\partial x}\left[\frac{\partial^{3} w}{\partial x^{3}}+\frac{\partial w}{\partial x}\left(\frac{\partial^{2} w}{\partial x^{2}}\right)^{2}+\frac{\partial^{3} w}{\partial x^{3}}\left(\frac{\partial w}{\partial x}\right)^{2}\right]-J \frac{\partial^{4} w}{\partial x^{2} \partial t^{2}} \\
+\frac{1}{2} \frac{\partial}{\partial x}\left\{\frac{\partial w}{\partial x} \int_{L}^{x} \rho A \frac{\partial^{2}}{\partial t^{2}}\left[\int_{0}^{x}\left(\frac{\partial w}{\partial x}\right)^{2} d x\right] d x\right\}=F_{E}-\rho A \frac{d^{2} y_{b}}{d t^{2}}
\end{gathered}
$$

where $w$ denotes the transverse displacement, $\rho$ is the mass density, $A$ is the cross-sectional area and $E$ is the modulus of elasticity. Here $I$ denotes the area moment of inertia with respect to the neutral axis, $J$ is the rotary inertia, $w_{b}$ is the base displacement due to ambient vibrations, and $F_{E}$ is the electrostatic force per unit length acting on the micro cantilever. The first and third terms on the left hand side of Eq. (1) account for translational and rotary inertias, respectively. The first part of the second term in the left hand side accounts for linear stiffness and the last part is due to curvature nonlinearities. Also, the last integral term in left hand side accounts for shortening effect due to inertial nonlinearities. The last term 
in right hand side accounts for translational inertia due to base excitation and is considered as a harmonic displacement in the form of:

$$
y_{b}=y_{0} \sin (\omega t)
$$

The electrostatic force between the electrodes of a parallel plate capacitor is given by [27]:

$$
F_{E}=\frac{d U_{E}}{d w}
$$

where $U_{E}$ is the electric potential energy stored in the capacitor and is given by [27]:

$$
U_{E}=\frac{1}{2} C V_{s}^{2}
$$

where $V_{S}$ is the surface voltage of the electret layer, and $C$ denotes the equivalent capacitance of the system which consists of two series capacitances including the electret layer capacitance $\left(C_{e}\right)$ and the variable capacitance $\left(C_{1}\right)$. To obtain the equivalent capacitance of the device, the capacitor is considered as an infinite number of differential parallel capacitors,

$$
d C_{1}(x, t)=\frac{\varepsilon_{0} b d x}{g_{d}-w(x, t)}
$$

each of them is in series with the electret layer capacitance.

$$
d C_{e}=\frac{\varepsilon_{0} \varepsilon_{e} b d x}{h_{e}}
$$

Neglecting the fringing effects and assuming complete overlap between the micro cantilever and the substrate, the equivalent differential capacitance of the system is obtained as follows:

$$
d C=\frac{\varepsilon_{0} b d x}{g_{d}+\frac{h_{e}}{\varepsilon_{e}}-w(x, t)}
$$

Integrating Eq. (7) over the length and substituting the resultant in Eq. (4), and using Eq. (3), the electrostatic force per unit length acting on the micro cantilever is obtained as follow:

$$
F_{E}=\frac{b \varepsilon_{0} V_{s}^{2}}{2\left(g_{d}+\frac{h_{e}}{\varepsilon_{e}}-w(x, t)\right)^{2}}
$$


Neglecting the effects of parasitic capacitances, the equivalent electric circuit of the energy harvester is modeled as Fig. 2.

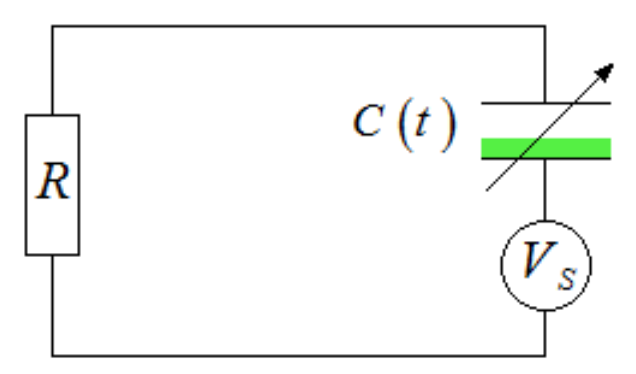

Fig. 2 The equivalent electric circuit of the electret-based capacitive energy harvester

Applying the Kirchhoff's voltage law in the equivalent circuit of the device, the electrical governing equation of the system, reduces to [3]:

$$
\frac{d Q}{d t}+\frac{Q}{R C}=\frac{V_{S}}{R}
$$

Eq. (1) along with Eq. (9) forms the nonlinear electro-mechanical governing equation of the system subjected to the following boundary conditions:

$$
\begin{array}{llll}
w=0, & \frac{\partial w}{\partial x}=0 & \text { at } & x=0 \\
\frac{\partial^{2} w}{\partial x^{2}}=0, & \frac{\partial^{3} w}{\partial x^{3}}=0 & \text { at } & x=L
\end{array}
$$

For convenience, the governing equations of the system are non-dimensionalized using following dimensionless parameters:

$$
\hat{x}=\frac{x}{L}, \quad \widehat{w}=\frac{w}{g_{d}}, \quad \hat{Q}=\frac{Q}{Q_{e}}, \quad \hat{t}=\frac{t}{T}, \quad \Omega=\omega T, \quad \hat{Q}=\frac{\mathrm{Q}}{\mathrm{Q}_{\mathrm{e}}}, \quad T=\sqrt{\frac{\rho A L^{4}}{E I}}
$$

where $Q_{e}$ is the electrical charge stored in electret layer, and is defined as:

$$
\mathrm{Q}_{\mathrm{e}}=\frac{\varepsilon_{0} \varepsilon_{e} b L}{h_{e}} V_{s}
$$

Introducing the dimensionless parameters given in Eq. (11) and the electrostatic force in Eq.(8) to Eq.(1) and Eq.(9) and removing the hat notation, the dimensionless governing equations reduces to: 


$$
\begin{gathered}
\frac{\partial^{2} w}{\partial t^{2}}+\frac{\partial}{\partial x}\left[\frac{\partial^{3} w}{\partial x^{3}}+\alpha_{1}\left(\frac{\partial w}{\partial x}\left(\frac{\partial^{2} w}{\partial x^{2}}\right)^{2}+\frac{\partial^{3} w}{\partial x^{3}}\left(\frac{\partial w}{\partial x}\right)^{2}\right)\right] \\
-\alpha_{2} \frac{\partial^{4} w}{\partial x^{2} \partial t^{2}}+\frac{\alpha_{1}}{2} \frac{\partial}{\partial x}\left\{\frac{\partial w}{\partial x} \int_{1}^{x} \frac{\partial^{2}}{\partial t^{2}}\left[\int_{0}^{x}\left(\frac{\partial w}{\partial x}\right)^{2} d x\right] d x\right\}=\frac{\alpha_{3} V_{s}^{2}}{\left(r_{1}-1\right)^{2}} \frac{Q^{2}}{\left(\int_{0}^{1} \frac{d x}{r_{1}-w}\right)^{2}\left(r_{1}-w\right)^{2}} \\
+Y_{b} \sin (\Omega t)
\end{gathered}
$$

and

$$
\frac{d Q}{d t}+\alpha_{4} \frac{Q}{\int_{0}^{1} \frac{d x}{r_{1}-w}}=\alpha_{5}
$$

where,

$$
\begin{gathered}
\alpha_{1}=\frac{g_{d}^{2}}{L^{2}}, \quad \alpha_{2}=\frac{J}{\rho A L^{2}}, \quad \alpha_{3}=\frac{b \varepsilon_{0} L^{4}}{2 E I g_{d}^{3}}, \quad r_{1}=1+\frac{h_{e}}{\varepsilon_{e} g_{d}}, \quad r_{2}=\frac{g_{u}}{g_{d}}, \quad \alpha_{4}=\frac{g_{d} T}{R b L \varepsilon_{0}}, \\
\alpha_{5}=\frac{h_{e} T}{R \varepsilon_{0} \varepsilon_{e} b L}, \quad Y_{b}=\frac{y_{0}}{g_{d}} \Omega^{2}
\end{gathered}
$$

and the boundary conditions in the non-dimensional form reduces to:

$$
\begin{aligned}
& w=0, \quad \frac{\partial w}{\partial x}=0 \quad \text { at } \quad x=0 \\
& \frac{\partial^{2} w}{\partial x^{2}}=0, \quad \frac{\partial^{3} w}{\partial x^{3}}=0 \quad \text { at } \quad x=1
\end{aligned}
$$

The Galerkin decomposition method is employed to eliminate the spatial dependence in Eq. (13) and Eq. (14). To this end, the transverse deflection of the micro-cantilever is represented as a series expansion in terms of the Eigen-functions of the micro-cantilever, i.e.

$$
w(x, t)=\sum_{i=1}^{N} U_{i}(t) \varphi_{i}(x)
$$

where $U_{i}(t)$ is the $i$ th generalized coordinate and $\varphi_{i}(x)$ is the $i$ th linear undamped mode shape of the micro-cantilever. Based on Galerkin method, the reduced order model of the system is obtained as given in appendix. The mean output power of the energy harvester $P$ is obtained based on the amplitude of the steady state response as: 


$$
P=\frac{1}{t_{2}-t_{1}} \int_{t_{1}}^{t_{2}} R\left(\frac{d Q}{d t}\right)^{2} d t
$$

Due to the surface voltage of the electret layer, it is necessary to calculate the static pull-in voltage of the micro-cantilever. To this end, the time-dependent terms in Eq. (13) are eliminated and the static behavior of the micro-cantilever is given by:

$$
\frac{\partial}{\partial x}\left[\frac{\partial^{3} w}{\partial x^{3}}+\alpha_{1}\left(\frac{\partial w}{\partial x}\left(\frac{\partial^{2} w}{\partial x^{2}}\right)^{2}+\frac{\partial^{3} w}{\partial x^{3}}\left(\frac{\partial w}{\partial x}\right)^{2}\right)\right]=\frac{\alpha_{3} V_{s}^{2}}{\left(r_{1}-w\right)^{2}}
$$

Using the Galerkin method, the static deflection and the pull-in voltage of the energy harvester is determined.

\section{Energy Conservation}

To clarify the problem and examine the energy conservation, we have developed the following equivalent electro-mechanical circuit for the reduced order model which accounts for the output energy through the electret layer and the pumped energy throughout the base excitation. A part of this energy is harvested in the resistance and the remaining is stored in terms of kinetic and potential energies in the vibration system and the potential energy in the variable gap capacitor.

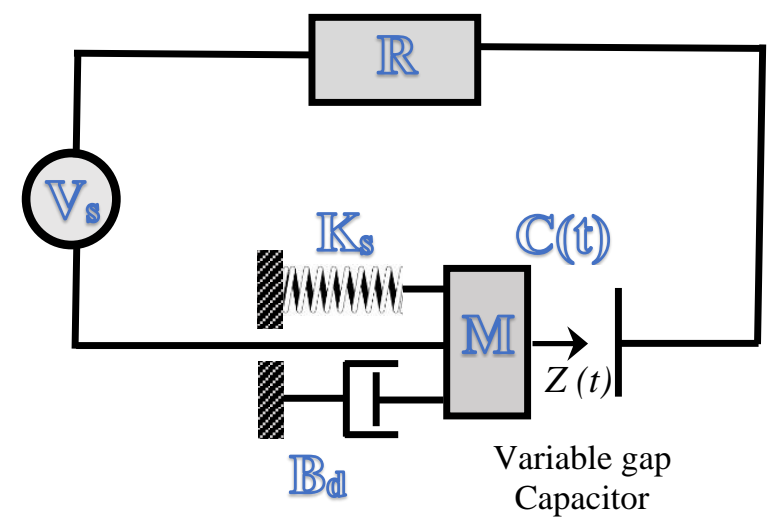

Fig. 3 The equivalent electro-mechanical circuit for energy conservation examination

Eq. (20) represents the energy conservation where the left-hand side corresponds to input energies and the right-hand side represents the harvested, mechanical and dissipated energies. 


$$
Q V_{s}+W_{e x}=\int_{0}^{t}\left(\frac{\partial Q}{\partial t}\right)^{2} R d t+\frac{1}{2} \frac{Q^{2}}{C(t)}+E_{m}+\int_{0}^{t} B_{d} \dot{Z}(t)^{2} d t
$$

where:

$$
E_{m}=\frac{1}{2} M \dot{Z}(t)^{2}+\frac{1}{2} K_{s} Z(t)^{2}
$$

In Eq. (20), $W_{e x}$, is the input energy due to the base excitation, $Q V_{s}$ represents the output energy from the electret layer, $E_{m}$ is the mechanical energy of the vibratory system and the last term in Eq. (20) denotes the dissipated energy due to mechanical damping. $M, K_{s}, B_{d}$, stand for the equivalent mass, stiffness and damping coefficients of the vibratory system.

\section{Results and discussion}

The mechanical, electrical and geometrical properties of the harvester are given in Table 1. The harvester is assumed to be made of silicon and CYTOP electret layer with breakdown voltage of $90 \mathrm{kV} / \mathrm{mm}$ [28].

\begin{tabular}{ll} 
Table 1: Mechanical, electrical and geometrical properties of the studied energy harvester \\
\hline Length, $L(\mu \mathrm{m})$ & 100 \\
Width, $\mathrm{b}(\mu \mathrm{m})$ & 20 \\
Thickness, $h(\mu \mathrm{m})$ & 3 \\
Young's modulus, $E(\mathrm{GPa})$ & 169.2 \\
Air gap, $g_{d}(\mu \mathrm{m})$ & 3 \\
Air gap, $g_{u}(\mu \mathrm{m})$ & 3 \\
Electret layer thickness, $h_{e}(\mu \mathrm{m})$ & 2 \\
Density of Si beam, $\rho\left(\mathrm{kg} / \mathrm{m}^{3}\right)$ & 2330 \\
Surface voltage of electret layer, $V_{s}(\mathrm{~V})$ & 180 \\
Relative permittivity of electret layer, $\varepsilon_{e}$ & 2 \\
Permittivity of free space, $\varepsilon_{0}\left(\frac{\mathrm{C}^{2}}{\mathrm{~N} \cdot \mathrm{m}^{2}}\right)$ & $8.85 \times 10^{-12}$
\end{tabular}

Fig. 4 illustrates the dimensionless tip deflection of the cantilever beam versus the applied electret surface voltage. It is shown that for a definite surface voltage, there exists either one or three equilibrium positions. In case merely one equilibrium position is available, the eigenvalues of the Jacobian matrix 
evaluated at the equilibrium point are negative and accordingly the fixed point is stable. However, once there are three equilibrium positions, two of them are stable (only one of them is of physical interest as the second one lays outside the gap and hence is not physically possible) and the other one is unstable. As the surface voltage is increased, the stable and unstable positions approaches each other and they coincide on the so-called pull-in voltage where the system undergoes a saddle node bifurcation and beyond it, both of the equilibrium positions disappear.

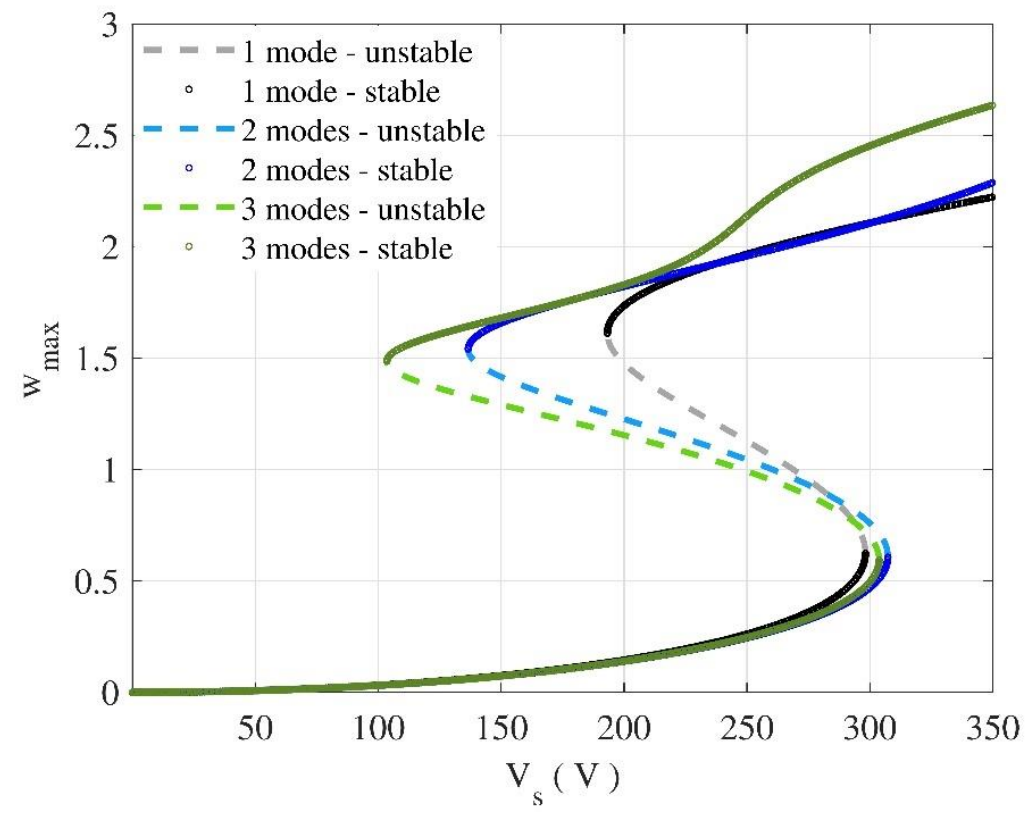

Fig. 4 The dimensionless deflection versus the electret surface voltage with the contribution of the first three vibrational modes.

As it is shown in Fig. 4, contribution of the first two modes in Eq. (17) provides a reasonable convergence and accordingly in the rest of the study the effect of the third and the higher modes in the system response are neglected. Fig. 5, depicts the time history and the phase diagram of the dynamic response of the energy harvester in the absence of the base excitation but exposed to initial disturbance. Here, the impetus is to examine the energy conservation by means of comparing the input energy with that captured throughout the output circuit. As depicted, once the initial disturbance is applied the output circuit, initiates transforming the mechanical energy into electrical and accordingly the motion amplitude dissipates [2]. 


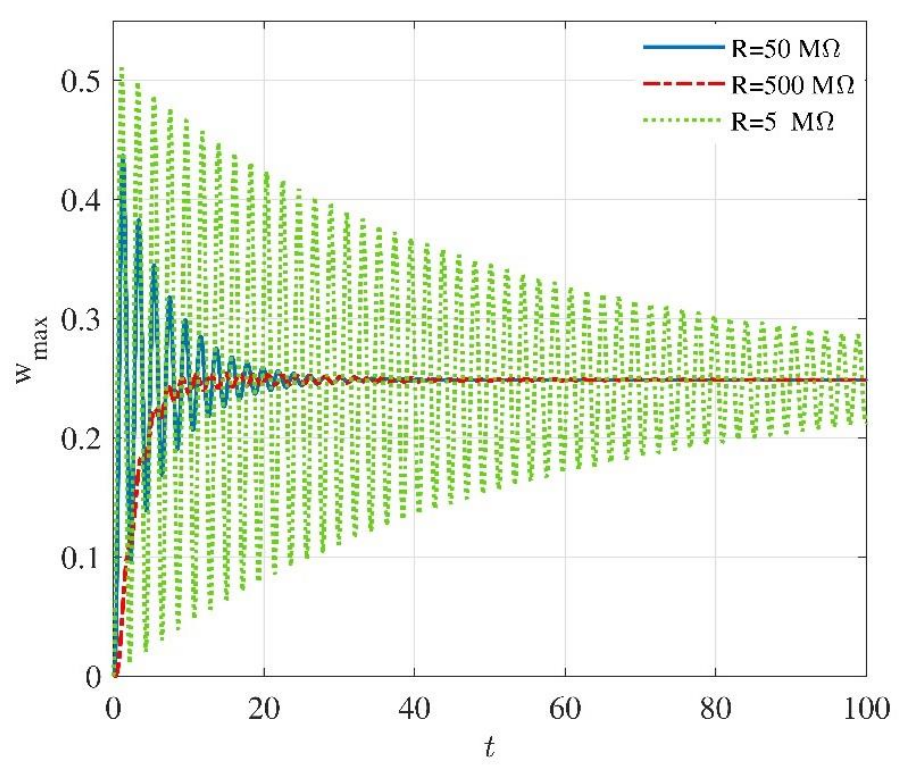

(a)

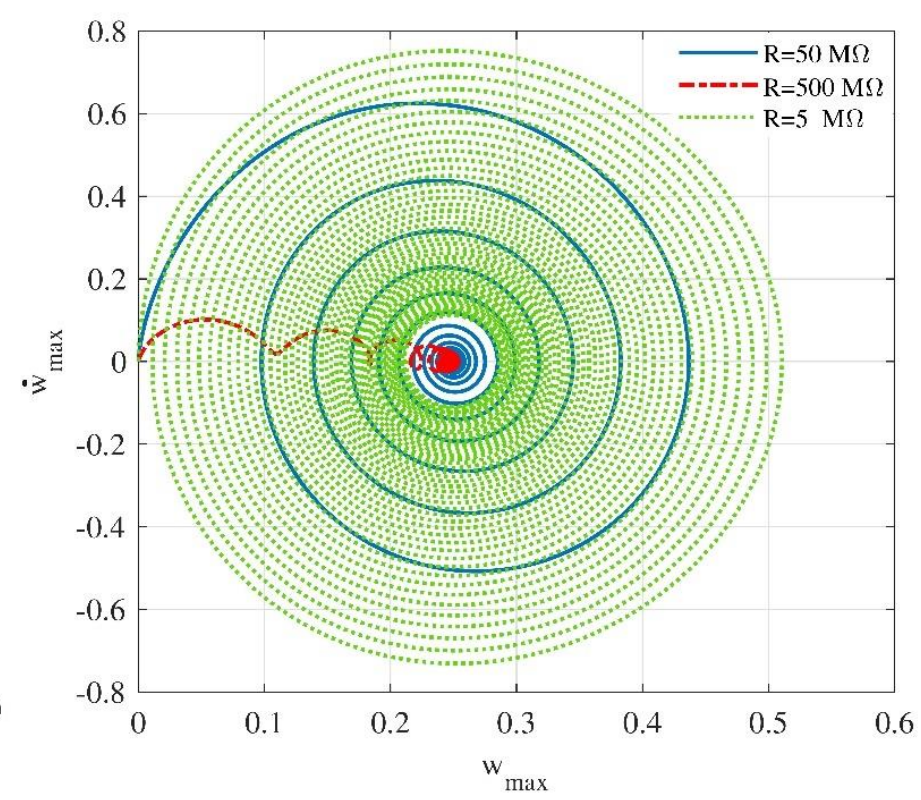

(b)

Fig. 5 The tip response, subjected to initial disturbance, in the absence of base excitation for $V_{s}=250(\mathrm{~V})$ a: time response, $b$ : phase plane

In the absence of base excitation, the energy conservation is examined by comparing the consumed and harvested energies throughout the output circuit. Once the circuit is closed due to the electrostatic force, the microbeam bends toward the electret layer and the electrical charge on both sides of the capacitor increases and accordingly an electric current flows in the circuit until the capacitor is charged. During this time span a part of the energy is harvested throughout the resistance. Table 2, gives the amount of consumed, harvested and stored energy in the capacitor for various electret layer voltages.

Table 2: The input and harvested energy for various output resistances and initial disturbances in the absence of base excitation

\begin{tabular}{|c|c|c|c|c|}
\hline$V_{s}(V)$ & $\begin{array}{c}Q V_{S} \\
\text { (Consumed energy) }\end{array}$ & $\begin{array}{c}\int_{0}^{t}\left(\frac{\partial Q}{\partial t}\right)^{2} R d t \\
\text { (Harvested energy) }\end{array}$ & $\begin{array}{c}\frac{1}{2} \frac{Q^{2}}{C(t)} \\
\text { (Stored energy in the capacitor) }\end{array}$ & $\begin{array}{c}\text { Efficiency (\%) } \\
\text { Harvested Energy }\end{array}$ \\
\hline 100 & $44.67 \mathrm{pJ}$ & $22.23 \mathrm{pJ}$ & $22.33 \mathrm{pJ}$ & 49.76 \\
\hline 150 & $101.80 \mathrm{pJ}$ & $50.32 \mathrm{pJ}$ & $50.90 \mathrm{pJ}$ & 49.42 \\
\hline 200 & $184.88 \mathrm{pJ}$ & $90.32 \mathrm{pJ}$ & $92.44 \mathrm{pJ}$ & 48.85 \\
\hline 250 & $299.56 \mathrm{pJ}$ & $143.21 \mathrm{pJ}$ & $149.78 \mathrm{pJ}$ & 47.80 \\
\hline
\end{tabular}

Fig. 6 depicts the time response, phase plane, and charge distribution on the capacitor plate for different electret layer voltages and $R=50 \mathrm{M} \Omega$. 


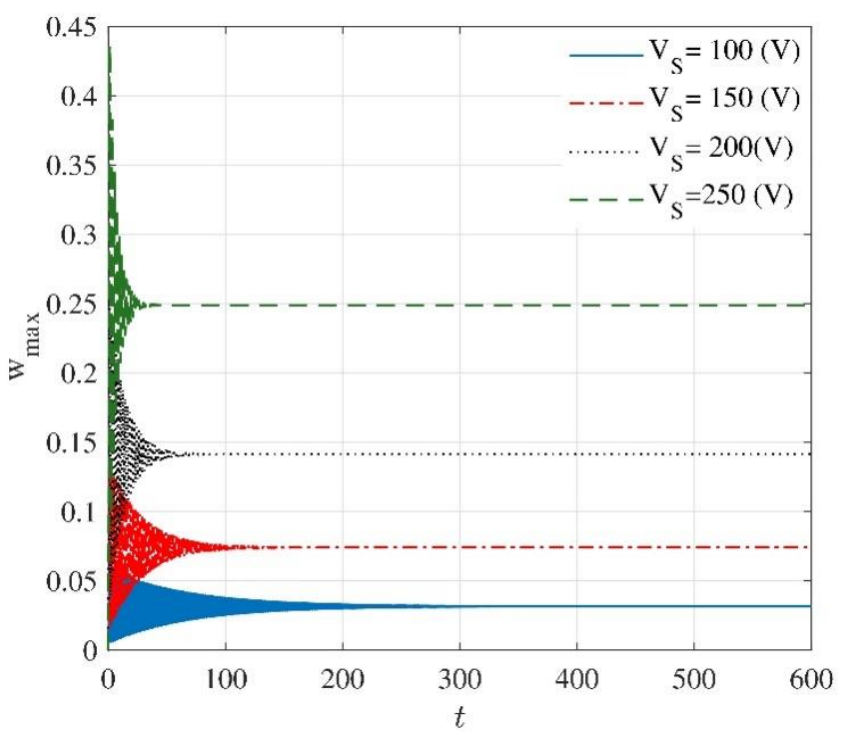

(a)

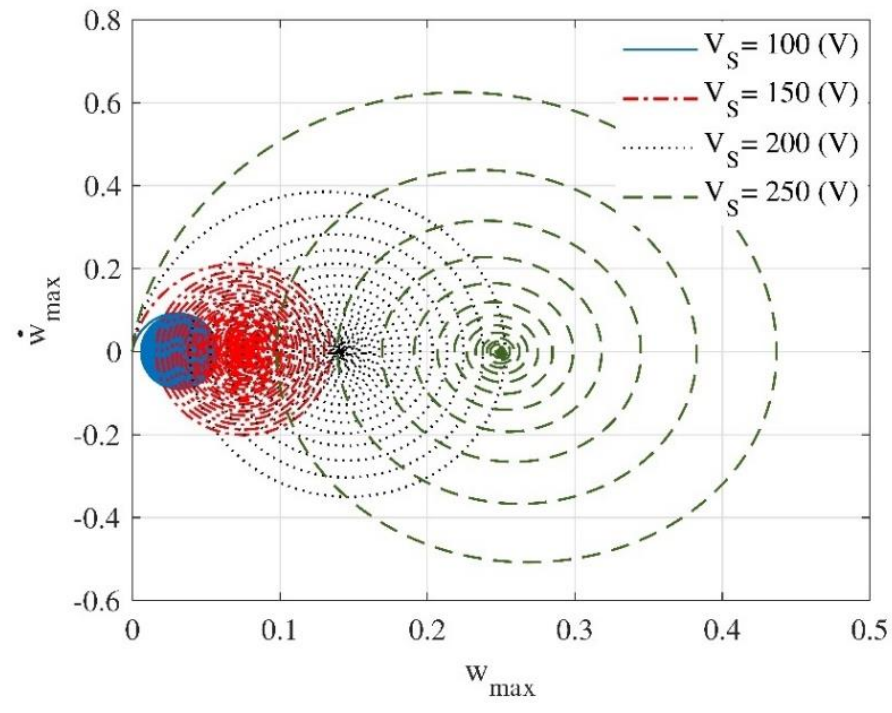

(b)

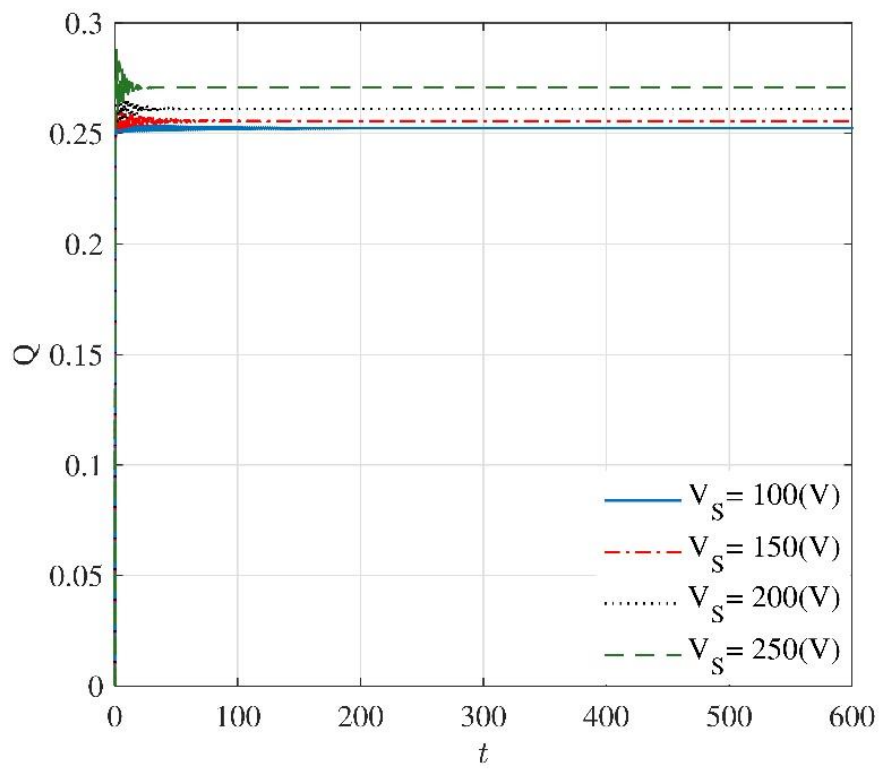

(c)

Fig. 6 The tip response, subjected to initial disturbance, in the absence of base excitation, (a): time response, (b): phase plane (c): Charge distribution on the capacitor (parameters are in non-dimensional form)

Considering the base excitation, Fig. 7 illustrates the harvested power for different load resistance with $250 \mathrm{~V}$ electret surface voltage. As we increase the electrical resistance the maximum harvested power increases up to a particular resistance $(R=15 \mathrm{M} \Omega)$ beyond which the harvested power decreases; this is because the effect of the higher electrical resistance on the harvested power is counterbalanced with the 
current reduction in the circuit and accordingly the harvested power exhibits a Lorenzian type behavior [2]. The solid and dashed lines represent the stable and unstable limit cycles. Capturing the unstable periodic solutions usually demands cumbersome iteration process as they have no attraction basins; some of the periodic solutions on the unstable branch have not been captured since they require a lot of iteration process.
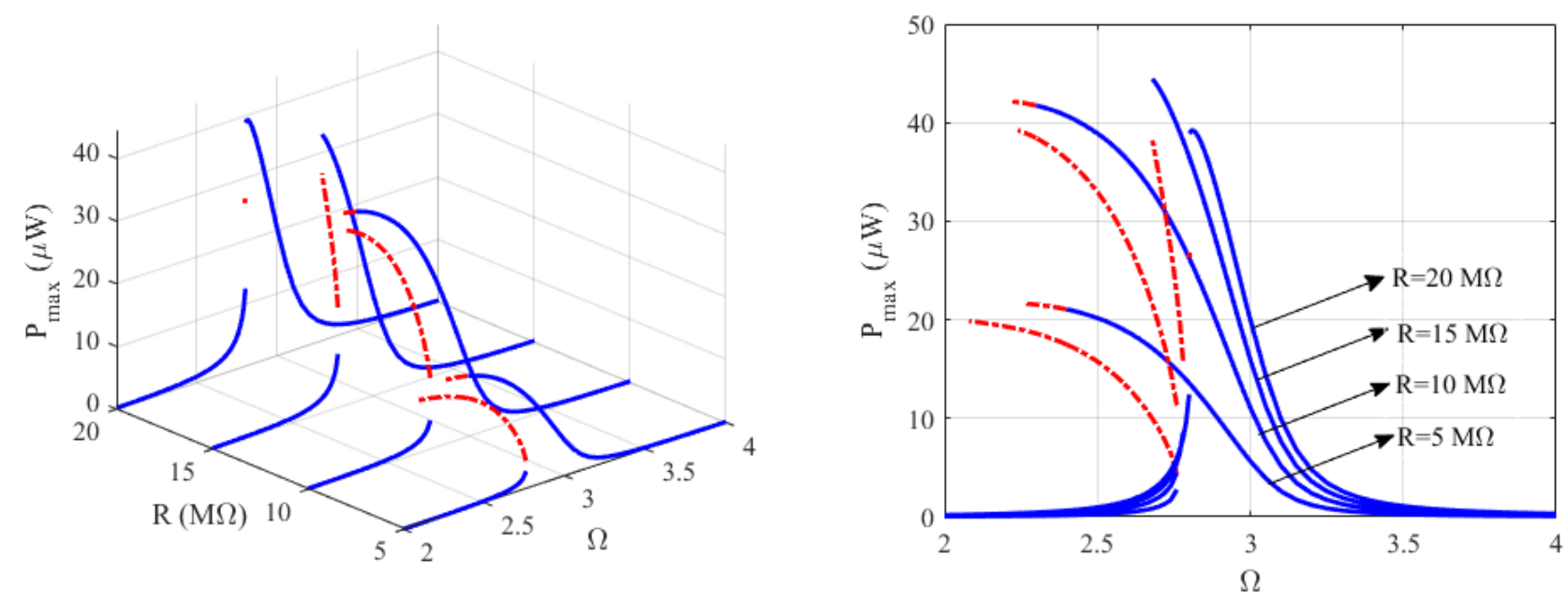

Fig. 7 The output power in the absence of mechanical damping for various resistances and $Y_{b}=0.15$

For a given $R$ as the frequency is swept forward the system response undergoes a jump to the higher amplitude periodic attractor in the cyclic fold bifurcation point where the branch of stable and unstable solutions collide and beyond it both of the branches disappear. As we sweep the frequency in the backward direction the response undergoes another jump to the lower amplitude periodic attractor in the period doubling bifurcation point where the Floquet multipliers leave the unit circle through -1 . The difference between the forward and backward frequency sweeps is regarded as hysteresis which is very common in the literature of nonlinear systems. In the region of multi-response solutions where the system exhibits more than one stable limit cycle, it is suggested that we push the system to vibrate on the highest amplitude limit cycle so that to harvest more energy; this is usually possible either by forward/backward frequency sweep or by disturbing the mass by an appropriate electrostatic initial voltage which pushes the system to the basin of the attraction of the desired limit cycle. Fig. 8 depicts the jumps in the time response which occur in the bifurcation points in both forward and backward frequency sweeps. 


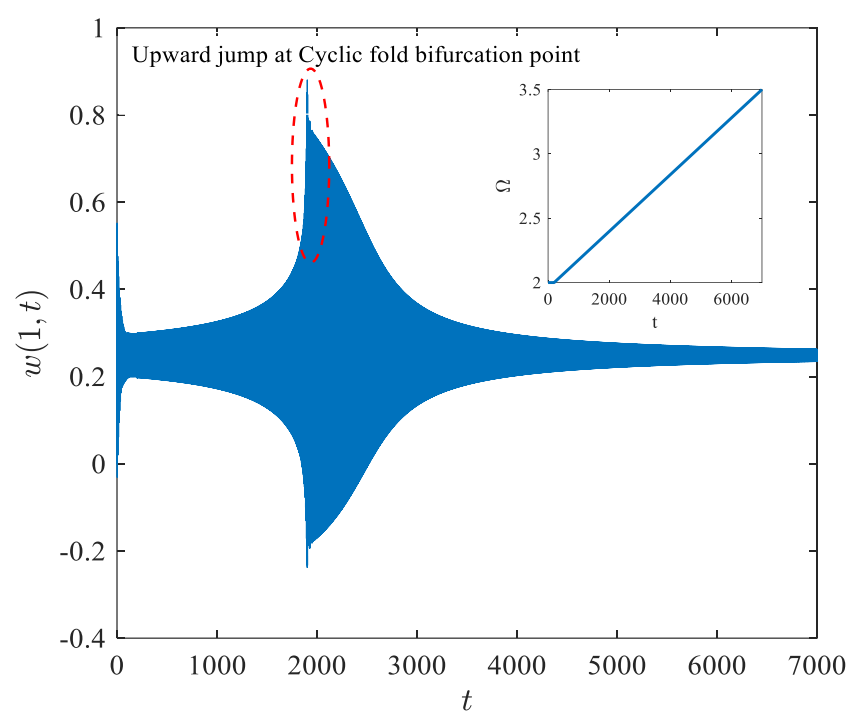

(a)

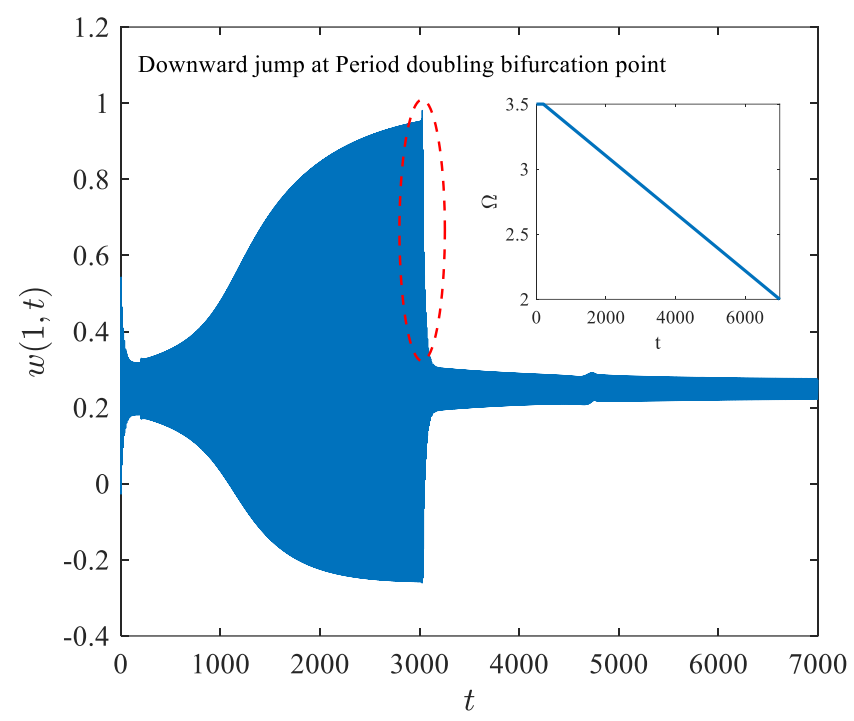

(b)

Fig. 8 The frequency sweep with $R=10 \mathrm{M} \Omega$ and $V_{S}=250 \mathrm{~V} ;(a)$ : forward sweep where upward jump takes place at the cyclic fold bifurcation point; (b): backward sweep where the downward jump takes place at period doubling bifurcation point.

Fig. 9, represents the frequency response curves for the same parameters as of Fig. 7. The results show that the system experiences higher damping effects for higher values of resistance. Therefore, by increasing the resistance, the nonlinear behaviour of the beam is reduced and the amplitude-frequency diagram of the beam straightens back to the right.
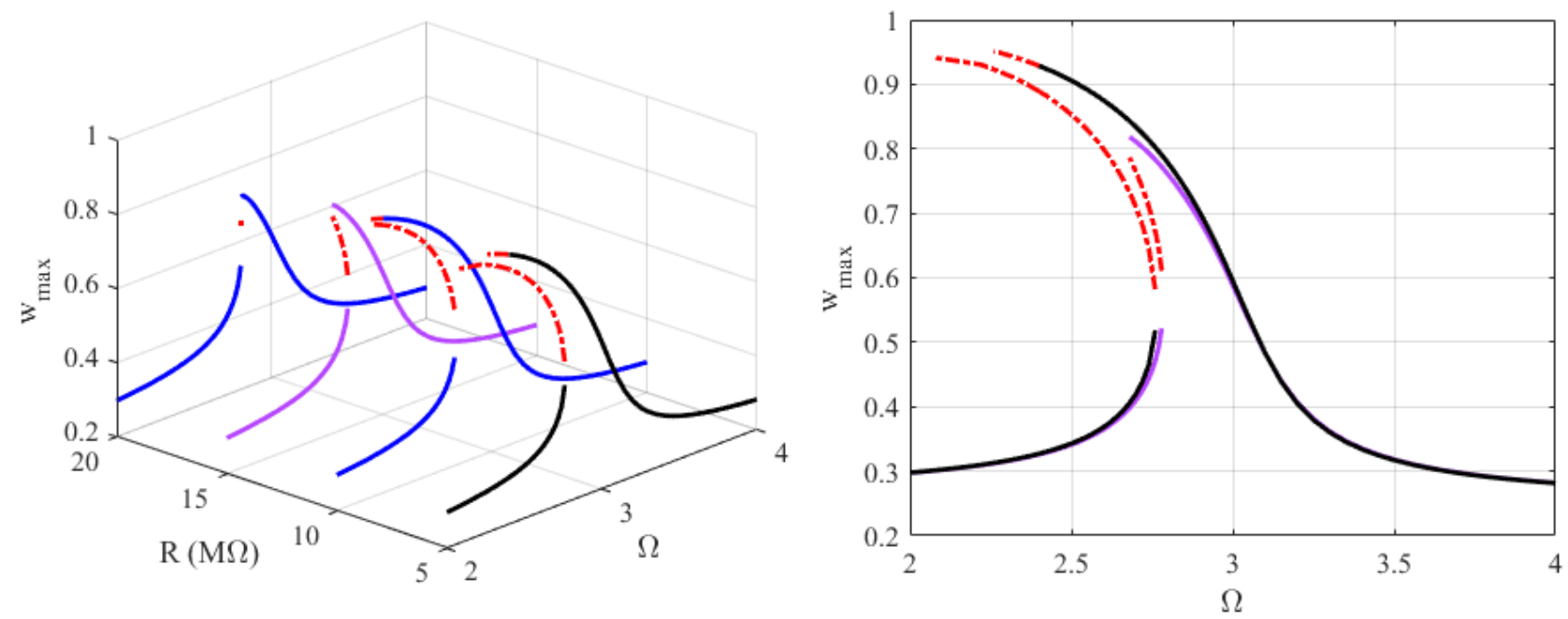

Fig. 9 The frequency response curves in the absence of mechanical damping for various resistances and $Y_{b}=0.15$

The more is the harvested power the less is the motion amplitude since more mechanical energy is extracted from the vibratory system. As the nonlinearity dominates the response, the bandwidth broadens 
and accordingly the harvested power within the bandwidth increases. Fig. 10 depicts the frequency response curves and the corresponding harvested powers for $R=15 \mathrm{M} \Omega, \mathrm{V}_{S}=250 \mathrm{~V}$ and different base excitation amplitudes.
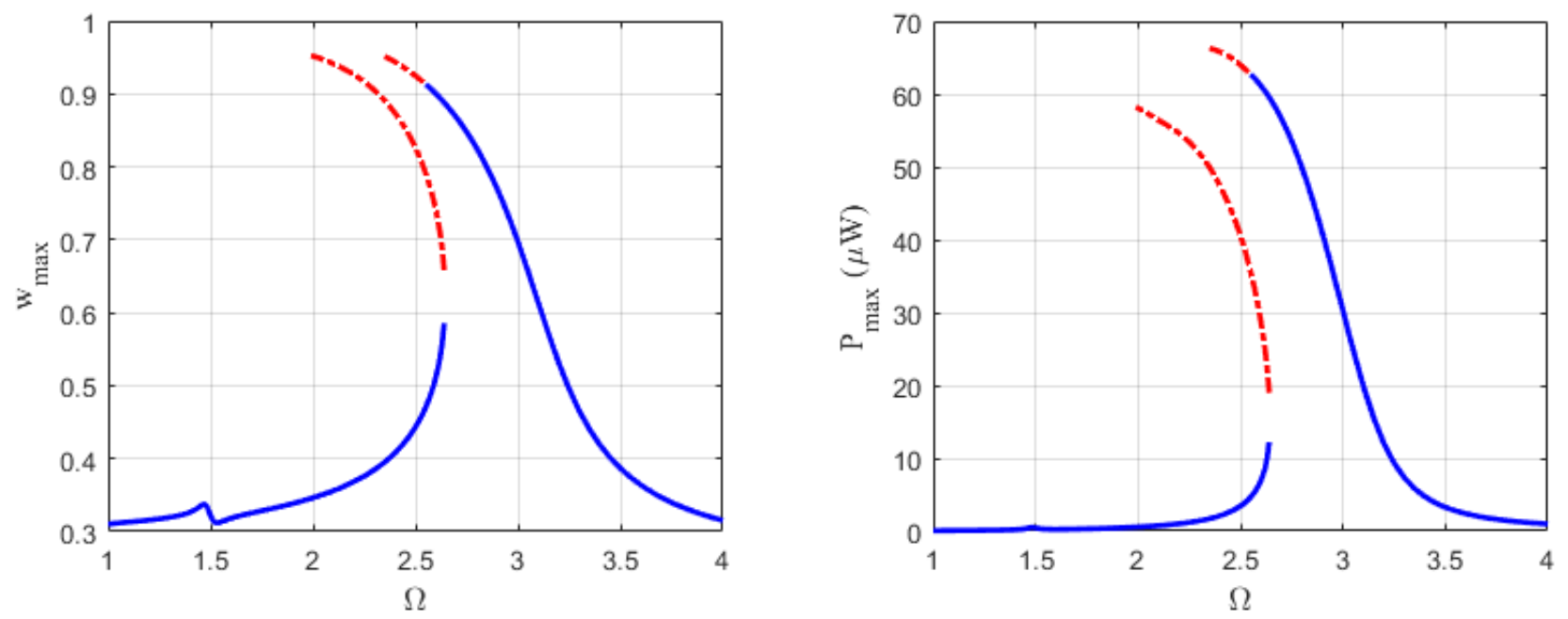

(a) $Y_{b}=0.3$
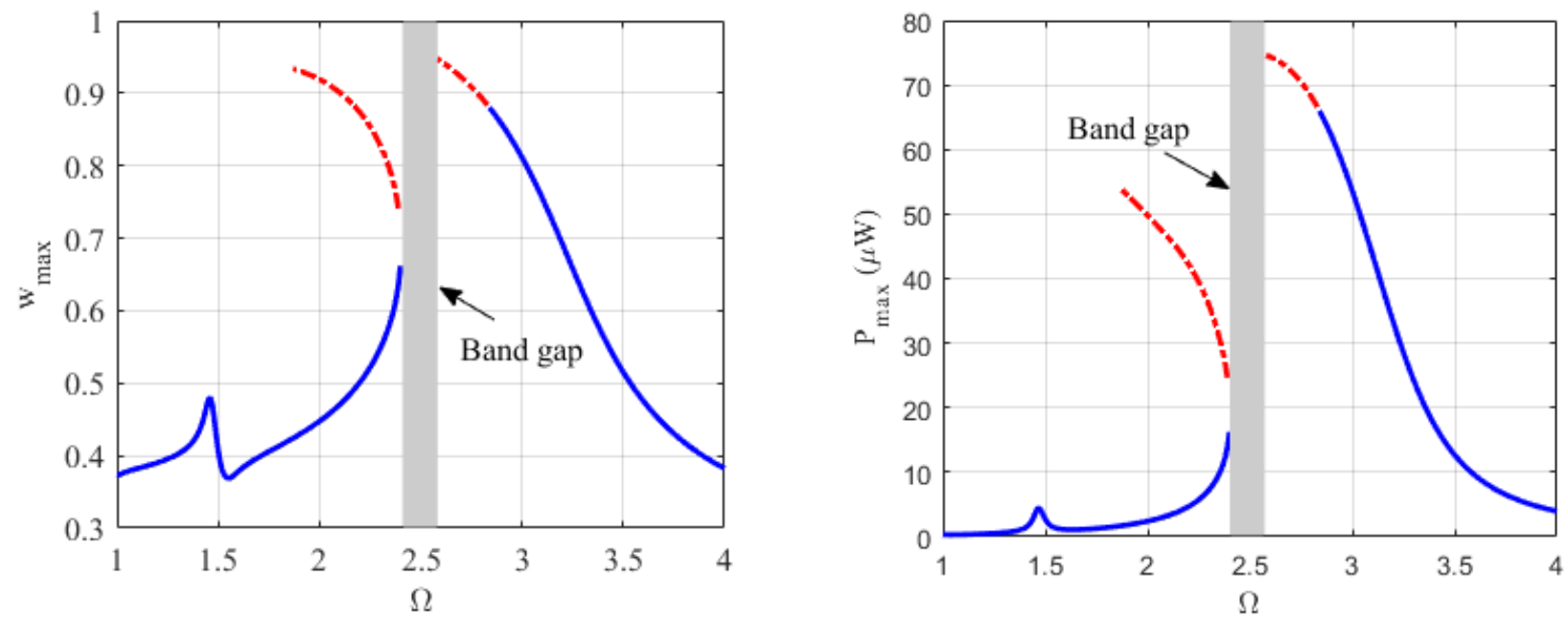

(b) $Y_{b}=0.6$ 

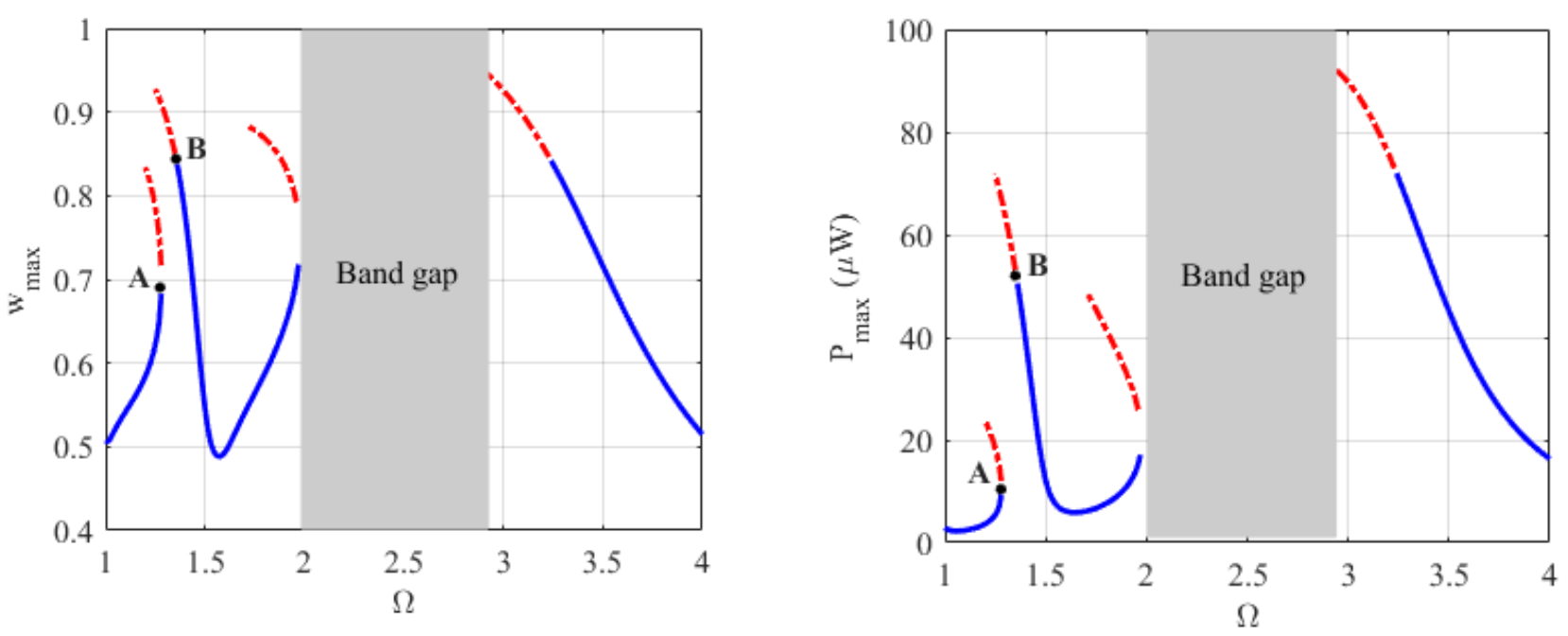

(d) $Y_{b}=1.2$
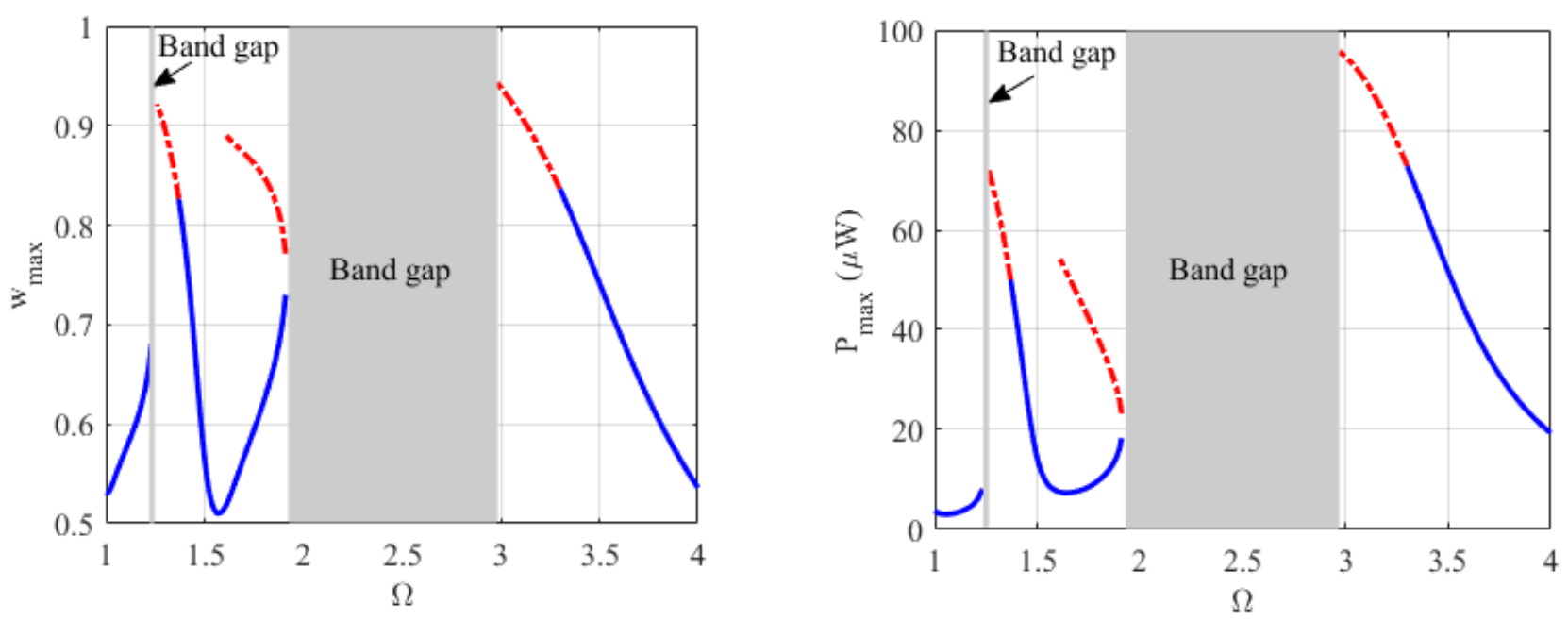

(e) $Y_{b}=1.3$ 

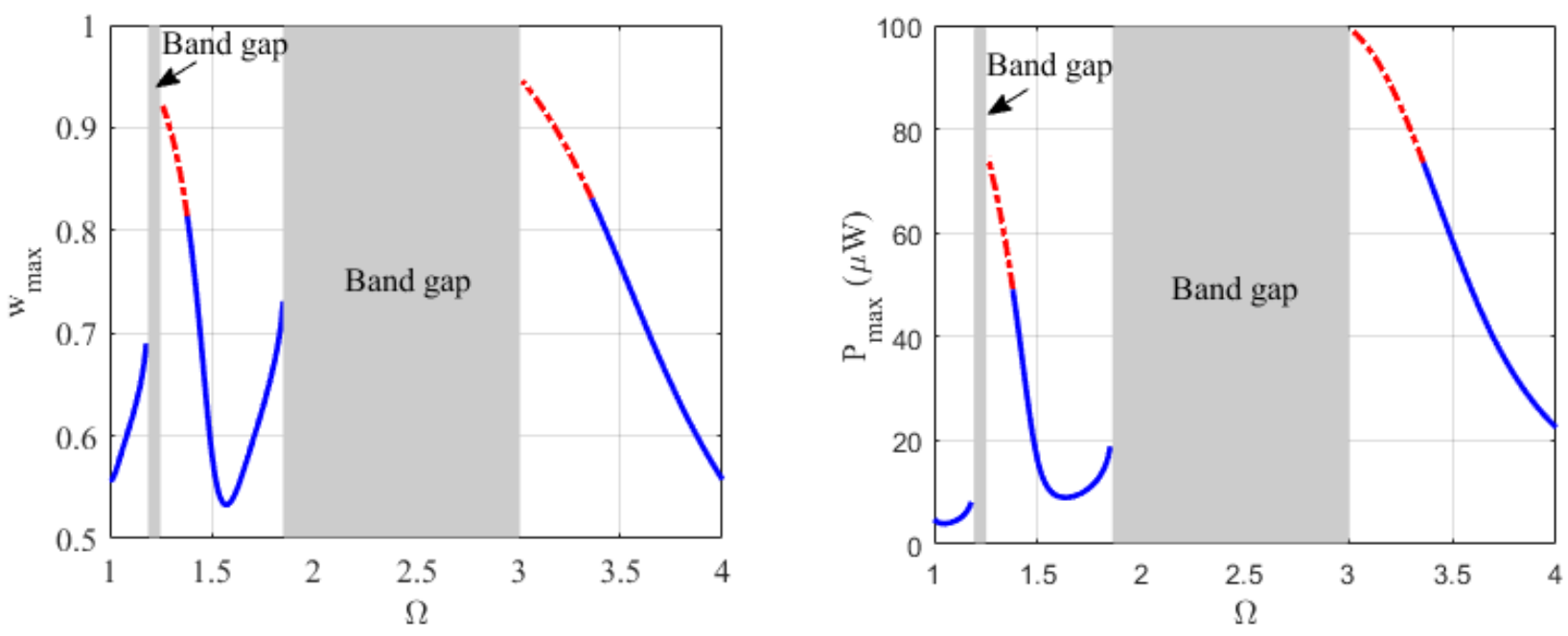

(f) $Y_{b}=1.4$
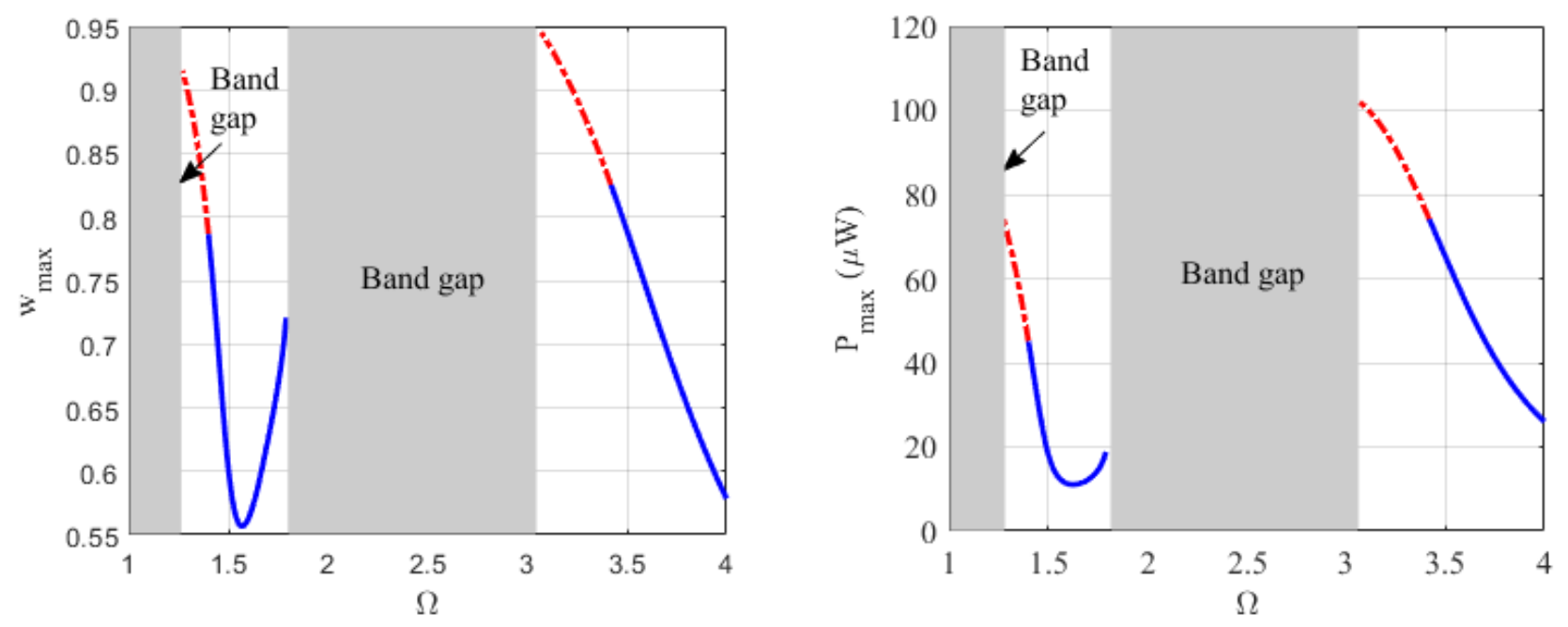

(g) $Y_{b}=1.5$

Fig. 10 The frequency response in the vicinity of the primary resonance for different base excitations at $R=15 \mathrm{M} \Omega$

As the base excitation amplitude increases the amplitude of the super-harmonic resonance of order two surges and accordingly the harvested power in this region rises. Further increase in the amplitude of the base excitation leads in the further energy harvesting as a result of the amplification of the motion amplitude in the super-harmonic resonance. For $Y_{b}=1.2$ the frequency response in the super-harmonic resonance region splits into two distinct parts and accordingly two more bifurcations including one cyclic fold (point A) and one period doubling bifurcation (point B) manifest. As we further increase the base excitation amplitude, band gap appears in the frequency response curve where pull-in occurs for any initial condition. For the energy harvesting application, once the device undergoes the excitation 
frequency in the vicinity of the super-harmonic resonance it is preferable for the system to be excited with the highest possible amplitude before the system undergoes band gap where pull-in occurs.

\section{Conclusion}

We proposed a nonlinear capacitive energy harvesting device which harvests the energy of the ambient mechanical vibrations. The nonlinearity sources are curvature nonlinearities, shortening effect and electrostatic force which result in the appearance of bifurcations, jumps, hysteresis and multi-response solution in the frequency response curves. The equations of motion were derived based on EulerBernoulli beam theory and discretized to a reduced order model by means of Galerkin method and the minimum required mode shapes to gain a reasonable converged response was determined. The periodic solutions were computed by shooting technique and their stabilities were examined based on the values of the corresponding Floquet multipliers. We inspected the energy harvesting enhancement in the multiresponse region which reveals a considerable increase in the ratio of the harvested energy to the input mechanical energy. The bifurcation types were studied on the frequency response curves and we reported upward and downward jump in the cyclic fold and period doubling bifurcation points in the forward and backward frequency sweeps respectively. We observed that as the amplitude of the base excitation increases the super-harmonic resonance of order two gets more pronounced and the harvested power increases considerably in the resonance region. As we further increased the base excitation amplitude, the amplitude of the limit cycles in the resonance region increased but a band gap immerged in the

frequency response curves where we could not capture any stable periodic attractors and accordingly any excitation in the band led to pull-in. We demonstrated that the nonlinearity of the response broadens the bandwidth which in turn enhances the energy harvesting.

\section{Declarations}

Conflict of interest: The authors declare that they have no conflict of interest.

\section{Data Availability}

Data sharing not applicable to this article as no datasets were generated or analysed during the current study. 


\section{References}

1. Marzencki, M., Defosseux, M. and Basrour, S., MEMS Vibration Energy Harvesting Devices With Passive Resonance Frequency Adaptation Capability. Journal of Microelectromechanical Systems, 2009. 18(6): p. 1444-1453.

2. Azizi, S., Ghodsi, A., Jafari, H., Ghazavi, M.R., A conceptual study on the dynamics of a piezoelectric MEMS (Micro Electro Mechanical System) energy harvester. Energy, 2016. 96: p. 495-506.

3. Ghavami, M., Azizi, S. and Ghazavi, M.R., On the dynamics of a capacitive electret-based micro-cantilever for energy harvesting. Energy, 2018. 153: p. 967-976.

4. Beeby, S.P., Torah, R. N., Tudor, M. J., Glynne-Jones, P., O'Donnell, T., Saha, C. R. and Roy, S. A micro electromagnetic generator for vibration energy harvesting. Journal of Micromechanics and Microengineering, 2007. 17(7): p. 1257-1265.

5. Türky1lmaz, S., Zorlu, Ö., Muhtaroğlu, A., Külah, H., An Electromagnetic Micro-Power Generator for Low Frequency Vibrations with Tunable Resonance. Procedia Engineering, 2011. 25: p. 729-732.

6. Belhaq, M. and Hamdi, M., Energy harvesting from quasi-periodic vibrations. Nonlinear Dynamics, 2016. 86(4): p. 2193-2205.

7. Abed, I., Kacem, N., Bouhaddi, N., Bouazizi, M. L., Multi-modal vibration energy harvesting approach based on nonlinear oscillator arrays under magnetic levitation. Smart Materials and Structures, 2016. 25(2): p. 025018.

8. Jafari, H., Ghodsi, A., Azizi, S.,Ghazavi, M.R., Energy harvesting based on magnetostriction, for low frequency excitations. Energy, 2017. 124: p. 1-8.

9. Aroudi, A.E., Ouakad, H., Benadero, L. and Younis, M., Analysis of Bifurcation Behavior of a Piecewise Linear Vibrator with Electromagnetic Coupling for Energy Harvesting Applications. International Journal of Bifurcation and Chaos, 2014. 24(5).

10. Dai, H.L., Abdelkefi, A. and Wang, L., Piezoelectric energy harvesting from concurrent vortex-induced vibrations and base excitations. Nonlinear Dynamics, 2014. 77(3): p. 967-981.

11. Mahmoudi, S., Kacem, N. and Bouhaddi, N., Enhancement of the performance of a hybrid nonlinear vibration energy harvester based on piezoelectric and electromagnetic transductions. Smart Materials and Structures, 2014. 23(7): p. 075024.

12. Firoozy, P., Khadem, S.E. and Pourkiaee, S.M., Broadband energy harvesting using nonlinear vibrations of a magnetopiezoelastic cantilever beam. International Journal of Engineering Science, 2017. 111: p. 113-133.

13. Madinei, H., Khodaparast, H. H., Friswell, M.I., Adhikari, S., Minimising the effects of manufacturing uncertainties in MEMS Energy harvesters. Energy, 2018. 149: p. 990-999.

14. Nie, X., Tan, T., Yan, Z., Yan, Z., Hajj, M. R., Broadband and high-efficient L-shaped piezoelectric energy harvester based on internal resonance. International Journal of Mechanical Sciences, 2019. 159: p. 287-305.

15. Zhu, J.X., Lin, J., Yuksek, N. S., Almasri, M., Feng, Z. C., Dynamic phenomena and analysis of MEMS capacitive power harvester subjected to low-frequency excitations. Nonlinear Dynamics, 2015. 79(1): p. 673688.

16. Boisseau, S., Despesse, G., Ricart, T., Defay, E. and Sylvestre, A., Cantilever-based electret energy harvesters. Smart Materials and Structures, 2011. 20(10): p. 105013.

17. Naruse, Y., Matsubara, N., Mabuchi, K., Izumi, M. and Suzuki, S., Electrostatic micro power generation from low-frequency vibration such as human motion. Journal of Micromechanics and Microengineering, 2009. 19(9): p. 094002.

18. Ibrahim, A., Ramini, A. and Towfighian, S., Experimental and theoretical investigation of an impact vibration harvester with triboelectric transduction. Journal of Sound and Vibration, 2018. 416: p. 111-124.

19. Liu, H. and Gao, X., Vibration energy harvesting under concurrent base and flow excitations with internal resonance. Nonlinear Dynamics, 2019. 96(2): p. 1067-1081.

20. Garg, A. and Dwivedy, S.K., Nonlinear dynamics of parametrically excited piezoelectric energy harvester with 1:3 internal resonance. International Journal of Non-Linear Mechanics, 2019. 111: p. 82-94. 
21. Tran, N., Ghayesh, M.H. and Arjomandi, M., Ambient vibration energy harvesters: A review on nonlinear techniques for performance enhancement. International Journal of Engineering Science, 2018. 127: p. 162185.

22. Lu, Z.-Q., Ding, H., and Chen, L.-Q., Resonance response interaction without internal resonance in vibratory energy harvesting. Mechanical Systems and Signal Processing, 2019. 121: p. 767-776.

23. Wu, H., Tang, L., Yang, Y., Soh, C. K., Development of a broadband nonlinear two-degree-of-freedom piezoelectric energy harvester. Journal of Intelligent Material Systems and Structures, 2014. 25(14): p. 18751889.

24. Wu, H., Tang, L., Yang, Y., Soh, C. K., A novel two-degrees-of-freedom piezoelectric energy harvester. Journal of Intelligent Material Systems and Structures, 2012. 24(3): p. 357-368.

25. Cammarano, A., Neild, S. A. Burrow, S. G. and Inman, D. J., The bandwidth of optimized nonlinear vibration-based energy harvesters. Smart Materials and Structures, 2014. 23(5): p. 055019.

26. Nayfeh, A. H. , Pai, P.F., Linear and Nonlinear Structural Mechanics. 2004: WILEY-VCH.

27. Cheng, D.K., Field and wave electromagnetics. 1989: Addison-Wesley Publishing Company.

28. Mohammad I, Y., MEMS linear and nonlinear statics and dynamics. 2011: Springer. 